\title{
Space Shuttle Thermal Protection System Repair Flight Experiment Induced Contamination Impacts
}

\author{
Kendall A. Smith*, Carlos E. Soares, Ron Mikatarian, Danny Schmidl \\ Boeing, Houston, TX, 77059 \\ Colin Campbell, Steven Koontz, Michael Engle \\ NASA JSC, Houston, TX, 77058 \\ and \\ Doug McCroskey, Jeff Garrett \\ Outgassing Services International, Mountain View, CA, 94043
}

\begin{abstract}
NASA's activities to prepare for Flight LF1 (STS-114) included development of a method to repair the Thermal Protection System (TPS) of the Orbiter's leading edge should it be damaged during ascent by impacts from foam, ice, etc.... Reinforced Carbon-Carbon (RCC) is used for the leading edge TPS. The repair material that was developed is named NonOxide Adhesive eXperimental (NOAX). NOAX is an uncured adhesive material that acts as an ablative repair material. NOAX completes curing during the Orbiter's descent. The Thermal Protection System (TPS) Detailed Test Objective 848 (DTO 848) performed on Flight LF1 (STS-114) characterized the working life, porosity void size in a micro-gravity environment, and the on-orbit performance of the repairs to pre-damaged samples. DTO 848 is also scheduled for Flight ULF1.1 (STS-121) for further characterization of NOAX onorbit performance. Due to the high material outgassing rates of the NOAX material and concerns with contamination impacts to optically sensitive surfaces, ASTM E 1559 outgassing tests were performed to determine NOAX condensable outgassing rates as a function of time and temperature. Sensitive surfaces of concern include the Extravehicular Mobility Unit (EMU) visor, cameras, and other sensors in proximity to the experiment during the initial time after application. This paper discusses NOAX outgassing characteristics, how the amount of deposition on optically sensitive surfaces while the NOAX is being manipulated on the pre-damaged RCC samples was determined by analysis, and how flight rules were developed to protect those optically sensitive surfaces from excessive contamination where necessary.
\end{abstract}

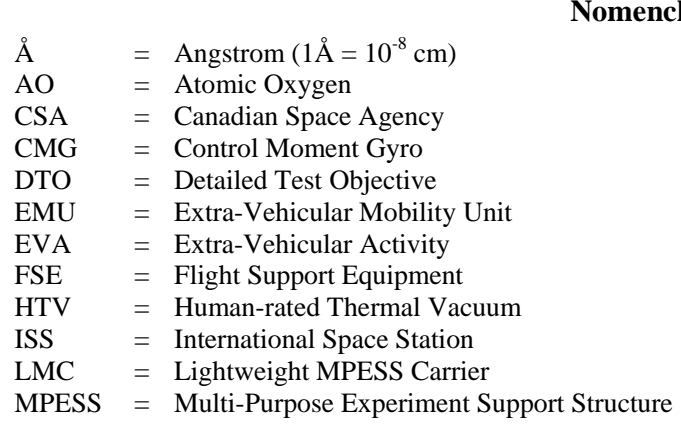

* Engineer/Scientist, Boeing-Houston, 13100 Space Center Blvd. HB3-20 .

1

American Institute of Aeronautics and Astronautics 


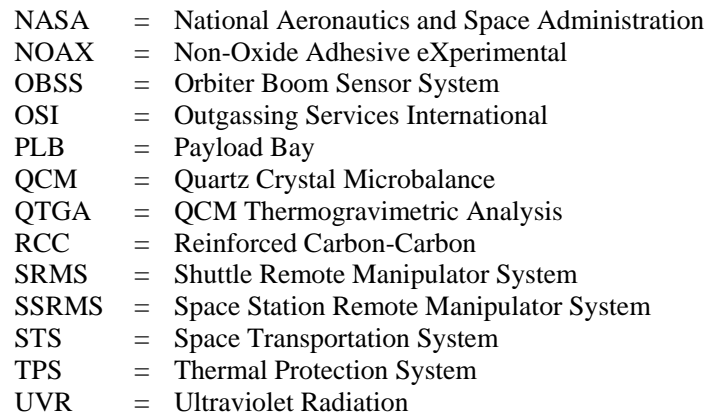

\section{Introduction}

S S part of NASA's preparation for Flight LF1 (STS-114), a method was developed to repair the Reinforced A Carbon-Carbon (RCC) on the leading edge of the Orbiter should it be damaged during ascent. The Non-Oxide Adhesive eXperimental (NOAX) material was developed to act as a cure-in-place ablative repair material [1]. The Thermal Protection System (TPS) Detailed Test Objective 848 (DTO 848) was performed on Flight LF1 (STS-114). A total of 3 pre-damaged RCC samples were flown for Flight LF1. Test objectives include evaluation of micro-gravity impacts on the material behavior during application and degassing, as well as, in the resultant porosity of hardened repairs. Additionally, evaluations were made with respect to hardened vacuum, sinusoidal temperature profiles, and attributes such as protuberance, step heights, ramp angles, surface roughness, micro-cracking and material layer thickness in the Low Earth Orbit (LEO) environment. DTO 848 is also scheduled for ULF1.1 (STS-121) to further characterize NOAX onorbit performance. Figure 1 shows the location of the experiment inside the Orbiter Payload Bay. Figure 2 shows the configuration of the RCC samples in the Lightweight Multi-Purpose Experiment Support

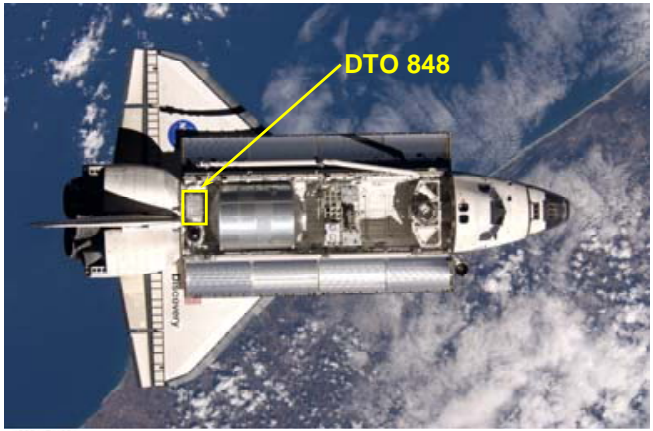

Figure 1: DTO 848 in Orbiter Payload Bay Structure (MPESS) Carrier (LMC) which is located in the back of the Orbiter Payload bay.

During Human-rated Thermal Vacuum (HTV) testing, where an astronaut performs a simulated repair in a large vacuum chamber, fogging of the Extra-vehicular Mobility Unit (EMU) visor was observed when dispensing NOAX onto RCC samples at a temperature of $49^{\circ} \mathrm{C}$ within a time period of 15 minutes. The EMU visor was estimated to be at a temperature of $-34^{\circ} \mathrm{C}$ while in the HTV due to the presence of liquid nitrogen cooled walls and the lack of a solar simulator. In contrast, thermal conditions on-orbit are known to be more benign, with the EMU visor operating in a temperature range of $-10^{\circ} \mathrm{C}$ to $16^{\circ} \mathrm{C}$. Removal of the deposited material using EVA wipes during HTV
testing was successful, however, there is a concern

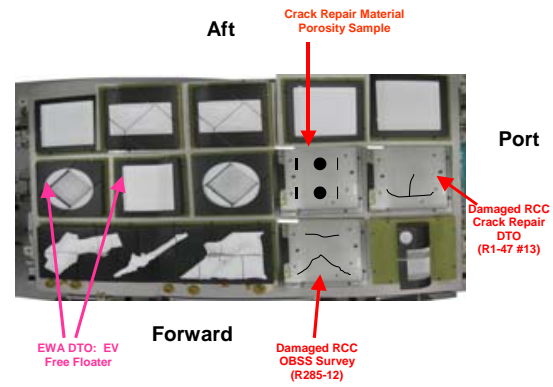

Figure 2: LF1 Configuration of LMC with RCC samples ${ }^{1}$

that when the contaminated visor is exposed to the on-orbit environment (Ultraviolet Radiation (UVR) and Atomic Oxygen (AO)), the material may fix to the surface of the visor and be difficult to remove. 
Due to the high material outgassing rates of the NOAX material and concerns about contamination sensitive surfaces (including the potential hazard of fogging of the EMU visor), ASTM E 1559 outgassing tests were performed to determine the condensable outgassing rate for NOAX as a function of time during the curing process[2]. During the first Flight LF1 EVA, NOAX was applied to one of three pre-damaged RCC samples. Another sample was used for an Orbital Boom Sensor System (OBSS) scan to evaluate the ability of the OBSS to detect damage on the RCC. The other samples were not used due to EVA time limitations.

\section{Materials Outgassing Induced Contamination}

Contaminant deposition and accumulation, in conjunction with exposure to the LEO environment (UVR and $\mathrm{AO}$ ), can produce degradation of the optical properties of contamination sensitive surfaces. Characterization and modeling of optical property degradation is crucial to the long-term operation and performance of equipment sensitive to optical degradation $[3,4,5]$.

ASTM E 1559 testing of ISS materials focus on outgassing rates toward the end of the six day test. Due to the comparatively short time period that the NOAX material will be on-orbit and the presence of sensitive surfaces, such as the EMU visor and OBSS optical sensors, during the first few hours of outgassing, it was critical to understand the initial outgassing rate behavior of the NOAX material. Additionally, this was the first known test of an uncured material in an ASTM E 1559 chamber. Special care had to be taken to ensure that the initial rate information could be accurately captured. Several samples of NOAX were supplied for ASTM E 1559 testing. A test duration of 48 hours was selected and the source and receiver temperatures were selected to afford comparison to ground test conditions and predicted on-orbit conditions. A QCM Thermogravimetric Analysis (QTGA) was performed to determine the nature and relative abundance of the outgassed species.

Composition analysis conducted during the QTGA indicated that the deposited material was composed primarily of several related complex organic or organic-silica compounds. The QTGA also confirmed the presence of a liquid deposit between temperatures of $-53^{\circ} \mathrm{C}$ and $-33^{\circ} \mathrm{C}$. This is an indirect observation based on the behavior of the QCM during the QTGA, actual range where liquid is present may be broader. The QTGA also demonstrated that not all of the deposited material was a permanent deposit. For a $100 \AA$ layer of contaminate, approximately $20 \%$ of the material deposited on the $-34^{\circ} \mathrm{C}$ QCM evaporated during a 5 hour period. For a $500 \AA$ layer, only $10 \%$ of the material evaporated during a 5 hour period. Following the isothermal period, the $-34^{\circ} \mathrm{C}$ QCM was heated at a rate of approximately $1^{\circ} \mathrm{C} / \mathrm{min}$. After reaching a temperature of $22^{\circ} \mathrm{C}$ most of the deposited material had evaporated. A conservative estimate of the evaporation rate during the warm up is $8 \AA / \mathrm{min}$.

\section{Non-Oxide Adhesive eXperimental Behavior}

During testing in the Human-rated Thermal Vacuum Chamber (HTV), profuse bubbling was observed particularly for NOAX applied at higher temperatures. In addition, condensation of material on the EMU visor was observed. This material was easily removed in the HTV, but on-orbit conditions, particularly with exposure to AO and UVR might cause the material to be difficult or impossible to remove on-orbit.

As can be seen in Figure 3, the first visible instance of fogging occurred after approximately 15 minutes of exposure to NOAX at $49^{\circ} \mathrm{C}$ with the visor at $-34^{\circ} \mathrm{C}$. Since NOAX was being dispensed continuously, the effective amount on the test surface during this period was approximately $8 \mathrm{in}^{2}$. Using the initial outgassing rate a deposit with an

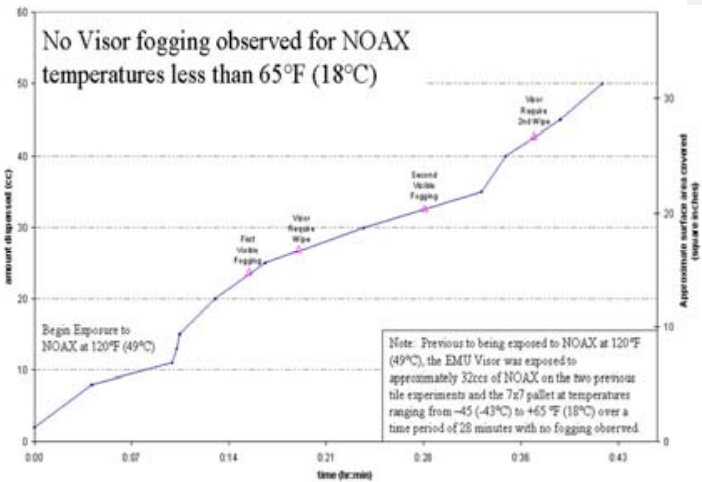

Figure 3: Timeline for dispensing of NOAX in HTV average thickness on the order of $30 \AA$ to $120 \AA$, respectively, would be expected due to the NOAX for the first instance of fogging in the timeline shown in Figure 3. It should be noted that a deposit of this thickness would not be expected to significantly impact the optical properties of the visor, therefore the deposit likely coalesced into droplets. The visor in the HTV had also already been exposed to a significant amount of NOAX at lower temperatures which may have also contributed to the fogging of the visor. 
During the on-orbit DTO, with the NOAX being at the nominal temperature of $27^{\circ} \mathrm{C}$, approximately $2 \AA$ to $8 \AA$ of material will deposit. If the temperature of the NOAX increases to $38^{\circ} \mathrm{C}$, then the amount of deposition would increase to between $7 \AA$ and $29 \AA$. If the temperature of the NOAX increases to $49^{\circ} \mathrm{C}$, then the amount of deposition would increase to between $11 \AA$ and $46 \AA$. For the nominal case with the NOAX at $27^{\circ} \mathrm{C}$, the amount of deposition is several orders of magnitude less than seen in the HTV and is highly unlikely to cause fogging of the visor.

The on-orbit DTO confirmed this prediction, as no fogging of the EMU visor was observed. Also, due to the zero gravity environment, bubbling did not occur, instead the material swelled slightly like rising bread. A swiping motion was discovered to work best to remove the volatile material from the NOAX to aid in application and curing. Figure 4 shows the NOAX after it has been applied during the DTO.

\section{ISS and Orbiter Sensitive Surfaces}

Outgassing contamination onto ISS optically sensitive surfaces is limited to $30 \AA / \mathrm{yr}$. ISS sensitive surfaces include the Lab window, and Space Station Remote Manipulator System (SSRMS) camera lenses. The SSRMS was used to observe the crew during the DTO and to perform operations in the Payload Bay which would place it in close proximity to the DTO including the removal of a replacement Control Moment Gyro (CMG) being delivered by the Orbiter. The hardware owner of the SSRMS, the Canadian Space Agency (CSA), agreed to allow up to $10 \AA$ of contamination on the lens for this DTO. The Lab window was to be open for brief

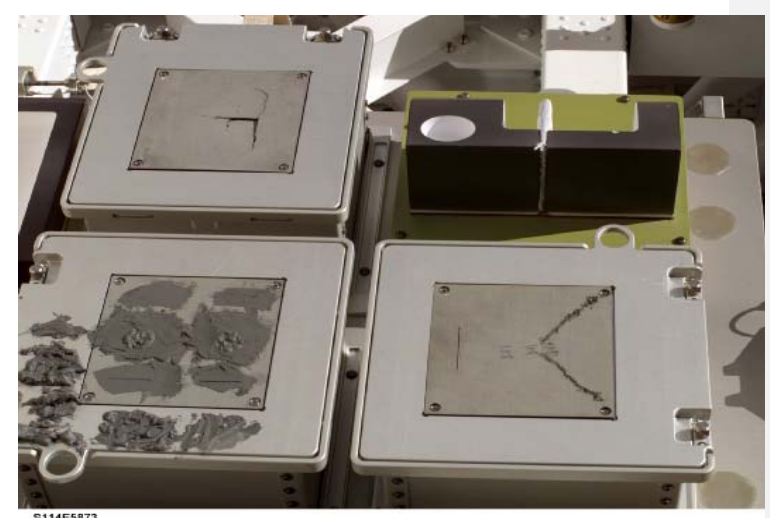

Figure 4: NOAX Application for DTO 848 periods of time for situational awareness. Since the Lab window is a science window with extreme sensitivity to deposited materials it was imperative to ensure that this surface receives minimal contamination.

Deposition onto Orbiter optically sensitive surfaces is less of a concern than ISS sensitive surfaces, since it is possible to clean or replace these surfaces when the Orbiter returns to Earth. Sensitive surfaces on the Orbiter include the Shuttle Remote Manipulator System (SRMS) cameras, the Orbiter Boom Sensor System (OBSS), and the Orbiter Payload Bay cameras.

Discussions with the Orbiter

Project Office resulted in a limit of $100 \AA$ of contamination for the SRMS cameras and the Orbiter Payload Bay cameras to prevent degradation of the use of these instruments while on-orbit. Since the OBSS is a highly sensitive instrument designed for analyzing of the Orbiter TPS it is desirable to maintain contamination levels on this instrument at minimal levels so an acceptable contamination level of $10 \AA$ was assigned to the OBSS. Planned operations for the OBSS included a scan of a damaged tile sample on the LMC 3 hours after the DTO has been performed. This would have placed the OBSS approximately $5 \mathrm{ft}$ away from the

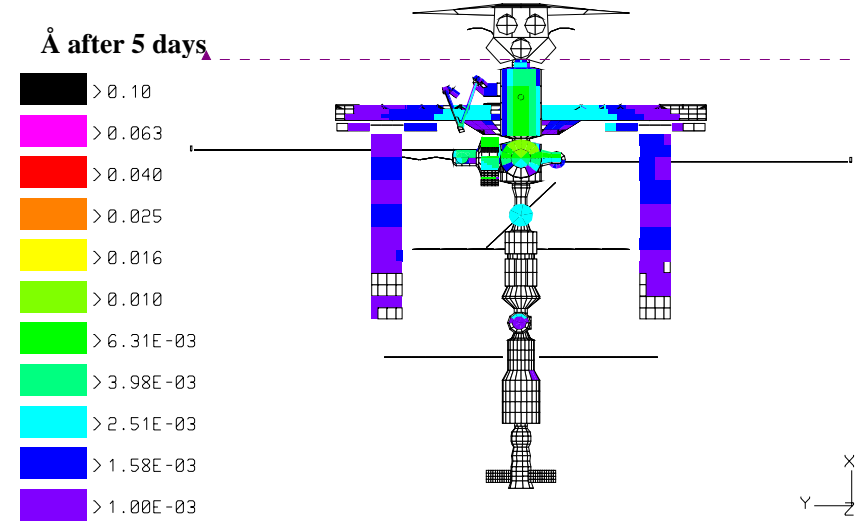

Figure 5: Deposition due to Orbiter DTO 848 on LF1 onto ISS after 5 days

American Institute of Aeronautics and Astronautics 
30 minutes. Only the aft port side Payload Bay camera was in a position to receive NOAX contamination as the other cameras were shielded by equipment or were too far from the DTO to be a concern.

As can be seen from Figure 5, less than an Angstrom of contamination was predicted for NOAX at the nominal temperature of $27^{\circ} \mathrm{C}$. These levels will have a negligible impact on the ISS system level requirements. If the nominal temperature of the NOAX increased to $49^{\circ} \mathrm{C}$, the deposition would still be negligible compared to the system level requirement. For the Lab Window, contamination due to the DTO will be a second or third order effect compared to the contribution of the Orbiter itself.

Flight rules and on-orbit support prevented the SSRMS cameras from exceeding their contamination limits.

Figure 6 details the levels of contamination that were predicted for the Payload Bay. If the Payload Bay camera looked directly at the DTO for the entire on-orbit duration, it would receive less than $30 \AA$ of contamination (most of which would be deposited in the first 6 hours). The SRMS would not exceed its limit of $100 \AA$ of contamination as long as it remained at least $3.5 \mathrm{ft}$ away from the DTO.

The OBSS is at highest risk of exposure to contamination when it performs a scan of a damaged RCC sample approximately 3 hours after the DTO has been performed. Analysis shows that the OBSS would receive less than $1 \AA$ of contamination during this half hour scan of the RCC sample for the nominal temperature of $27^{\circ} \mathrm{C}$. If the temperature increases to $38^{\circ} \mathrm{C}$ the amount of contamination received

\section{$\AA$ after 5 days}
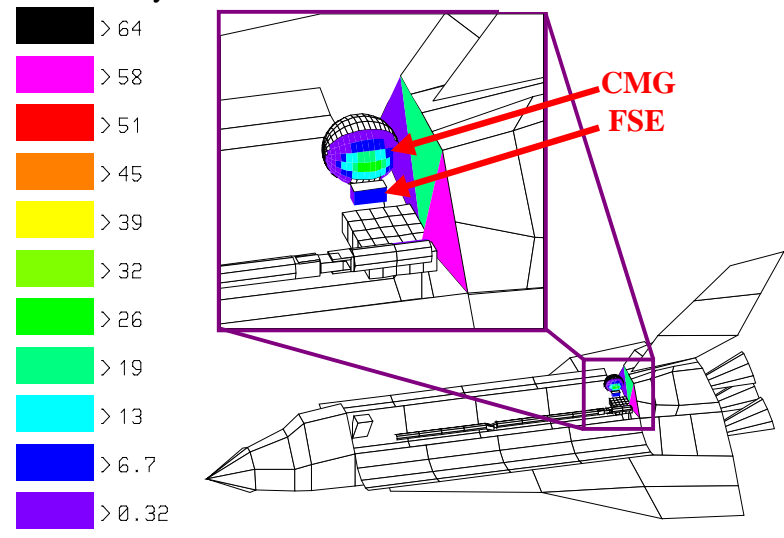

Figure 6: Angstroms of Contamination in Orbiter Payload Bay after 5 Days of Outgassing from NOAX on LF1 (STS-114) increases to $2.5 \AA$ for the half hour scan. The ISS attitude was constrained to maintain temperatures less than $38^{\circ} \mathrm{C}$.

\section{CONCLUSIONS}

No visor fogging was observed (as predicted) and no optical degradation of the OBSS sensors and the SSRMS/SRMS/PLB cameras occurred due to Flight LF1 (STS-114) DTO 848. It was also found that on-orbit temperatures were significantly lower than expected, resulting in over prediction of contaminant deposits.

In addition, the contamination observed in the HTV (EMU visor fogging) was successfully explained, and it was determined that conditions on-orbit would not cause fogging to occur.

Also, an uncured ablative repair compound was successfully tested for condensable outgassing rates, and the critical initial time data was utilized for analysis tasks. The analysis results were used to generate flight rules to mitigate impacts to the ISS, EMU and Orbiter optically sensitive surfaces, as required.

The analysis tools and techniques developed for ISS external contamination analysis were critical to modeling contamination transport. Techniques used for Flight LF1 DTO 848 will be used for Flight ULF1.1 DTO 848. New analyses will be conducted due to a substantial increase of NOAX usage and possible changes in the Orbiter Payload Bay thermal environment.

\section{REFERENCES}

${ }^{1}$ Master Development Test Plan for the RCC On-orbit Crack Repair Material (JSC-62487)

${ }^{2}$ ASTM, "Standard Test Method for Contamination Outgassing Characteristics of Spacecraft Materials, E 1559 03,” ASTM International, Oct. 2003. 
${ }^{3}$ Soares, C., Mikatarian, R. and Barsamian, H., "International Space Station Bipropellant Plume Contamination Model," $8^{\text {th }}$ AIAA/ASMT Joint Thermophysics and Heat Transfer Conference, AIAA 2002-3016, St. Louis, Missouri, 24-27 June 2002.

${ }^{4}$ Soares, C.E. and Mikatarian, R.R., "Understanding and Control of External Contamination on the International Space Station," Proceedings of the 9th International Symposium on Materials in a Space Environment, European Space Agency (ESA) publication SP-540, Noordwijk, The Netherlands, 2003.

${ }^{5}$ Boeder, P.A., Visentine, J.T., Shaw, C.G., Carniglia, C. K., Alred, J.W., Soares, C.E., "Effect of a silicone contaminant film on the transmittance properties of AR-coated fused silica", SPIE-04, 2004. 